\title{
Extracellular vesicles as novel carriers for therapeutic molecules
}

\author{
Nambin Yim $^{1}$ \& Chulhee Choi ${ }^{1,2,3, *}$ \\ ${ }^{1}$ Department of Bio and Brain Engineering, KAIST, ${ }^{2}$ Cellex Life Sciences Inc., ${ }^{3}$ Cancer Metastasis Control Center, KAIST Institute for the \\ Biocentury, KAIST, Daejeon 34141, Korea
}

Extracellular vesicles (EVs) are natural carriers of biomolecules that play central roles in cell-to-cell communications. Based on this, there have been various attempts to use EVs as therapeutic drug carriers. From chemical reagents to nucleic acids, various macromolecules were successfully loaded into EVs; however, loading of proteins with high molecular weight has been huddled with several problems. Purification of recombinant proteins is expensive and time consuming, and easily results in modification of proteins due to physical or chemical forces. Also, the loading efficiency of conventional methods is too low for most proteins. We have recently proposed a new method, the so-called exosomes for protein loading via optically reversible protein-protein interaction (EXPLORs), to overcome the limitations. Since EXPLORs are produced by actively loading of intracellular proteins into EVs using blue light without protein purification steps, we demonstrated that the EXPLOR technique significantly improves the loading and delivery efficiency of therapeutic proteins. In further in vitro and in vivo experiments, we demonstrate the potential of EXPLOR technology as a novel platform for biopharmaceuticals, by successful delivery of several functional proteins such as Cre recombinase, into the target cells. [BMB Reports 2016; 49(11): 585-586]

*Corresponding author. E-mail: cchoi@kaist.ac.kr

https://doi.org/10.5483/BMBRep.2016.49.11.174

Received 10 October 2016

Keywords: Drug delivery, Exosome, Extracellular vesicles, Light-induced protein-protein interaction, Protein drug

Abbreviations: CIB1, CRY-interacting basic-helix-loop-helix 1; CIBN, a truncated version of CIB1; CRY2, cryptochrome 2; EV, Extracellular vesicles; EXPLOR, Exosomes for protein loading via optically reversible protein-protein interaction; MVB, Multivesicular bodies

Perspective to: Nambin Yim et al (2016) Exosome engineering for efficient intracellular delivery of soluble proteins using optically reversible protein-protein interaction module. Nature communications, 22;7:12277. doi: 10.1038/ncomms 1227
All multicellular organisms develop various ways for exchange of various biomolecules, including not only small chemicals, but also nucleic acids and proteins. As major carriers of biomolecules, extracellular vesicles (EVs) play central roles in intercellular communication. EVs are spherical nano-size particles surrounded by a lipid bilayer, and further classified based on the physical and biochemical properties. Representatively, microvesicles derived from plasma membrane are $100-1,000 \mathrm{~nm}$, and exosomes originating from intracellular multivesicular bodies (MVBs) are 50-200 nm in size. EVs can be simply isolated by ultracentrifugation, size exclusion chromatography or ultrafiltration, and can be easily distinguished by primarily size, and by detecting the surface markers such as tetraspanins in secondary screening.

EV-based drug delivery was originally attempted for small chemical drugs such as curcumin, a well-known anti-inflammatory drug, after loading into exosomes (Dongmei Sun et al. Molecular therapy, 2010; 18(9); 1606-1614. doi: 10.1038/ mt.2010.105). EV-based drug delivery has been shown to solve several major problems, such as non-specific biodistribution and short half-life of drugs in the systemic circulation. Drug-loaded EVs can be produced by simple incubation of EVs with chemical drugs at room temperature, and various drugs, including doxorubicin, porphyrins, and paclitaxel, were successfully loaded and delivered by EV. The first biopharmaceutical for EV-based delivery was a short interfering RNA (Lydia Alvarez-Erviti et al. Nature biotechnology. 2011; 29(4); 341-345. doi:10.1038/nbt.1807). The authors transferred siRNAs into exosomes using an electroporation method, and showed that the nucleic acids-loaded exosomes can deliver siRNAs to the brain in mice. Since then, not only siRNA but also various nucleic acids such as microRNAs, messenger RNAs and plasmid DNAs, have been successfully loaded into the exosomes and delivered in vitro and in vivo.

Simultaneously, several methods for intracellular protein delivery using EVs have also been tried (Fig. 1). Unlike chemical drugs or nucleic acids, proteins cannot passively penetrate cellular membranes. Furthermore, proteins are easily modified by the physical and chemical environment, limiting the applicability of EV-based delivery of proteins with therapeutic potentials. In 2011, one group reported that highly oligomeric proteins can be targeted to exosomes by plasma

ISSN: 1976-670X (electronic edition)

Copyright (C) 2016 by the The Korean Society for Biochemistry and Molecular Biology

(c) This is an open-access article distributed under the terms of the Creative Commons Attribution Non-Commercial License (http://creativecommons.org/licenses/by-nc/4.0) which permits unrestricted non-commercial use, distribution, and reproduction in any medium, provided the original work is properly cited. 


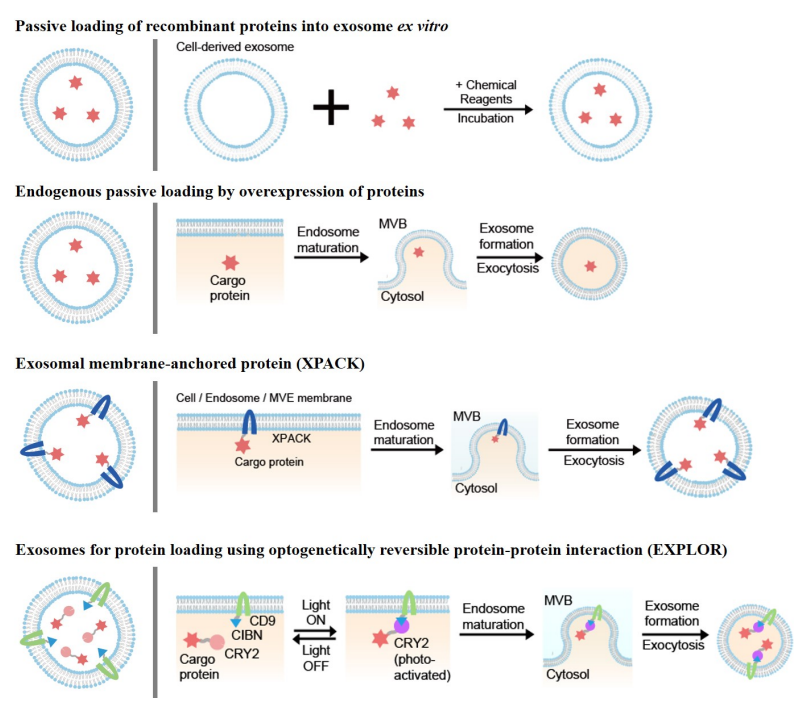

Fig. 1. Methods for loading proteins into exosomes. Passive loading of recombinant proteins into exosome ex vitro: recombinant proteins can be loaded into exosomes by various methods such as simple incubation, freeze-thawing, sonication, or extrusion. Endogenous passive loading by overexpression of proteins: proteins are passively loaded into exosomes in exosome, producing cells overexpressing target proteins with low possibilities. Exosomal membrane-anchored protein (XPACK): proteins are attached to the inner layer of exosomal membrane, thus can be conjugated with exosomes along natural exosome biogenesis. Exosomes for protein loading using optogenetically reversible protein-protein interaction (EXPLOR): proteins are actively loaded into exosomes under light illumination. The interaction being reversible, the proteins get detached from the exosome membrane as free forms in the lumen of exosomes.

membrane anchors (Shen B et al. J Biol Chem. 2011; 286; 14383-14395. doi: 10.1074/jbc.M110.2086600). Based on this principle, they developed the EV targeting technology, and finally commercialized the method now called as XPACK technology (System Biosciences, Mountain View, CA, USA). The caveat of this method is that targeting proteins should be anchored to the exosome membrane, thus limiting the subcellular localization of delivered proteins to the cellular membrane of the recipient cells. Another group recently proposed alternative ex vitro methods for passive loading of recombinant proteins into exosomes (Matthew J. Haney et al. Journal of Controlled Release. 2015; 207; 18-30. doi:10.1016/ j.jconrel.2015.03.033). They tried to load catalases into exosomes using various methods, including simple incubation, freeze-thaw, sonication, and extrusion, and demonstrated successful delivery of recombinant intracellular proteins. Since these loading processes were based on mechanical dispersion, this method cannot be applied for the therapeutic potential of unstable proteins.

Exosomes for protein loading via optically reversible protein-protein interaction (EXPLORs) are novel protein carriers developed for overcoming the limitations of conventional EV-based protein delivery. The EXPLOR technology was designed such that the protein drug can be actively and transiently docked into the exosomes by blue light illumination. For that, cryptochrome 2 (CRY2), a photoreceptor of Arabidopsis thaliana that can bind to CRY-interacting basic-helix-loop-helix 1 (CIB1) by blue light wavelength, was conjugated to intracellular protein drug, and the exosomeassociated tetraspanin protein CD9 was conjugated to CIBN (a truncated form of $\mathrm{CIB} 1$ ). Once the cargo proteins are introduced into the exosomes via endogenous biogenesis, they can be detached from CD9-conjugated CIBN by removal of the illumination source, resulting in their release into the intraluminal space of the exosomes and efficient delivery to the cytosolic compartment of target cells. Thus, not only does the EXPLOR technology show a dramatic increase of the loading efficiency in exosomes compared to the previous protein loading systems (such as passive loading of recombinant proteins into exosomes ex vitro or XPACK), but it also shows a high efficiency of cytosolic protein delivery. Specifically, we successfully demonstrate the phenotype changes in various cell types and in the loxP-STOP-loxPeNpHR3.0-eYFP transgenic mice with EXOPLORs loaded with Bax, super-repressor IKB (a S32A and S36A mutant form of $\mathrm{I} \kappa \mathrm{B})$ and Cre recombinase.

In conclusion, we have recently developed a novel EV-based protein carrier EXPLOR that can deliver therapeutic proteins efficiently in vitro and in vivo, using active targeting induced by blue light. Since EXPLOR does not require the steps for purifying proteins, it is simple and cost-effective. Additionally, because EXPLOR adopts an endogenous active protein loading process instead of exogenous passive loading, most of the intracellular proteins, such as transcription factors, signal transducers, and enzymes, can be efficiently targeted for EXPLOR-based therapeutics. These advantages will overcome the limitations of the previous protein drug delivery, opening a new paradigm for future biopharmaceuticals.

\section{ACKNOWLEDGEMENTS}

This research was supported by KAIST Institute for the BioCentury, Cancer Metastasis Control Center (Grant Number: N11160071). 